\title{
Markov Chain Monte Carlo Bayesian Analysis for Population Pharmacokinetics of Dasatinib in Japanese Adult Subjects with Chronic Myeloid Leukemia and Philadelphia Chromosome Positive Acute Lymphoblastic Leukemia
}

\author{
Hiroyuki YOSHITSUGU*1, Yasuhiko IMAI*2, Taku SERIU*2 \\ and Masaki HIRAOKA*2 \\ *1 Discovery Medicine \& Clinical Pharmacology, Research \& Development, Bristol-Myers Squibb, New Jersey, USA \\ *2 R\&D Japan, Bristol-Myers K.K., Tokyo, Japan
}

\begin{abstract}
This study evaluated the population pharmacokinetics of dasatinib in Japanese subjects with imatinibresistant or -intolerant chronic myeloid leukemia (CML) and Philadelphia chromosome positive acute lymphoblastic leukemia $\left(\mathrm{Ph}^{+} \mathrm{ALL}\right)$ enrolled in 3 Japanese clinical trials, and compared individual PK estimates to that of non-Japanese subjects in global database. A full Markov Chain Monte Carlo Bayesian analysis method in NONMEM 7 was utilized for the estimation of model parameters. In terms of covariate model selection, baseline demographic and clinical laboratory covariates were assessed on oral clearance $(\mathrm{CL} / \mathrm{F})$. The food effect and the dose effect were also tested on the relative bioavailability $\left(F_{R}\right)$ and $\mathrm{CL} / \mathrm{F}$, respectively. A total of 706 observations were obtained from 63 Japanese subjects who received twice daily administration of dasatinib at 50 , 70 and $90 \mathrm{mg}$, and once daily administration at $100 \mathrm{mg}$. Consistent with a PPK model in non-Japanese subjects, plasma concentration-time data were well described by a linear two-compartment model with the inter-occasion variability (IOV) on the relative bioavailability $\left(\mathrm{F}_{\mathrm{R}}\right)$, which is to account for between-visit difference of dasatinib exposure within a subject apparently observed in a phase $1 / 2$ study in Japanese subjects. Comparable exposures of individual Japanese subjects with that of non-Japanese subjects were obtained. Investigation of covariates revealed neither marked trends nor clinically relevant effect on $\mathrm{CL} / \mathrm{F}$ or $\mathrm{F}_{\mathrm{R}}$. These results indicated that no dose adjustment is warranted for Japanese $\mathrm{CML}$ and $\mathrm{Ph}^{+} \mathrm{ALL}$ patients, based on their body size, age, or gender.
\end{abstract}

Key words : dasatinib, population pharmacokinetics, diagnostic statistics, DIC, MCMC Bayesian analysis

\section{Introduction}

Dasatinib ( SPRYCEL $\left.^{\circledR}\right)$ is a potent, broad spectrum ATP-competitive inhibitor of 5 critical oncogenic tyrosine kinases/kinase families : BCR-ABL, SRC, cKIT, PDGFR, and ephrin receptor kinases ${ }^{1,2}$. In particular, the BCR-ABL tyrosin kinase is associated with chronic myeloid leukemia (CML) and dasatinib differs from imatinib in that it can bind to both the active and inactive conformations of the ABL kinase domain $^{3-5)}$. Early- and late-phase clinical trials demonstrated dasatinib are efficacious and well tolerated in subjects with all phases of CML and Philadelphia chromosome positive acute lymphoblastic leukemia $\left(\mathrm{Ph}^{+} \mathrm{ALL}\right)$ in patients resistant or intolerant to imatinib, and in patients with newly diagnosed chronic phase $\mathrm{CML}^{6-19)}$. Dasatinib was approved by the US Food and Drug Administration (FDA) and the European Medicines Agency (EMA) for the treatment of imatinib-resistant or -intolerant CML and $\mathrm{Ph}^{+} \mathrm{ALL}$ and newly diagnosed chronic phase CML with a starting dose of $100 \mathrm{mg}$ or $140 \mathrm{mg}$ once daily (QD). In Japan, dasatinib was approved by Ministry of Health, Labor and Welfare (MHLW) for the treatment of imatinib-resistant or -intolerant CML and $\mathrm{Ph}^{+} \mathrm{ALL}$ and newly diagnosed CML with a starting

Address for Correspondence : YOSHITSUGU H. Oncology/Immunology group, Discovery Medicine \& Clinical Pharmacology, Research \& Development, Bristol-Myers Squibb, 3551 Lawrenceville Road Princeton, NJ 08648, US (Mailing Address : P. O. box 4000 Princeton, NJ 08543-4000, US)

(Express : Manuscript received July 11, 2011 ; revised December 5, 2011 ; accepted December 22, 2011) 
dose of $100 \mathrm{mg}$ QD or $70 \mathrm{mg}$ twice a day (BID $)^{1,20)}$.

Several clinical pharmacology studies have been conducted to investigate the PK of dasatinib ${ }^{1,6-10,20)}$. $\mathrm{C}_{\max }$ of dasatinib are observed between 0.5 and 6 hours following oral administration. Dasatinib exhibits dose proportional increases in AUC and linear elimination characteristics over the dose range of 15 $\mathrm{mg}$ to $240 \mathrm{mg} /$ day. The overall mean terminal halflife of dasatinib is $3-5$ hours. Data from a study of 54 healthy subjects administered a single, $100 \mathrm{mg}$ dose of dasatinib under fed conditions resulted in a $14 \%$ increase in the mean AUC of dasatinib, suggesting that the food effect is not clinically relevant. Preclinical studies showed that the major enzyme responsible for the metabolism of dasatinib is CYP3A4 and coadministration of dasatinib with ketoconazole increased exposure of dasatinib by approximately 4to 5 -fold. Conversely, the exposure of dasatinib was decreased by approximately $80 \%$, when it was coadministered with rifampin. Dasatinib PK also depends on gastric $\mathrm{pH}$ since the drug's solubility decreases as $\mathrm{pH}$ increases. Administration of Maalox ${ }^{\circledR}$ (aluminum hydroxide/magnesium hydroxidecontaining antacid) 2 hours prior to the dose of dasatinib caused an increase of approximately $26 \%$ in $\mathrm{C}_{\max }$ and had no effect on AUC. When Maalox was coadministered, both $\mathrm{C}_{\max }$ and AUC were reduced by $58 \%$ and $55 \%$, respectively, compared to when dasatinib was administered alone. Also, the $\mathrm{C}_{\max }$ and AUC values of dasatinib 10 hours following the famotidine dose were reduced by approximately $63 \%$ and $61 \%$, respectively, compared to when dasatinib was administered alone.

In a population pharmacokinetic (PPK) analysis in non-Japanese patients with $\mathrm{CML}$ and $\mathrm{Ph}^{+} \mathrm{ALL}$, the extent of dasatinib exposure was characterized by a linear two-compartment model with first-order absorption $^{21,22)}$. None of baseline demographic and clinical laboratory covariates was found to be clinically-relevant on the apparent clearance of dasatinib. Furthermore, individual dasatinib exposures obtained by applying the PPK model were used to characterize exposure-response (E-R) relationships with respect to safety (pleural effusion) and efficacy endpoints (major cytogenetic response: MCyR) in imatinib-resistant or -intolerant chronic phase CML patient population. The results of E-R analyses indicated that steady-state time-averaged plasma concentration $\left(\mathrm{C}_{\text {avg ss }}\right)$ and steady-state trough level $\left(\mathrm{C}_{\min \text { ss }}\right)$ are key parameters for the efficacy and the safety, respectively, using the data from a phase 3 study (Study CA180-034).

PPK analyses based on a non-linear mixed effect model were increasingly performed in drug development and the most popular software for these analyses is NONMEM which was updated to version 7 in 2009 with new estimation methods including Iteration Two Stage (ITS) and Markov Chain Monte Carlo (MCMC) Bayesian (BAYES) methods ${ }^{23)}$. In previous version of NONMEM, the first-order conditional estimation (FOCE) with interaction is preferably used in practice to appropriately estimate model parameters, particularly when the data is rich and model not too non-linear in terms of parameters. However this method is an approximated, linearized optimization method which may often introduce bias into the maximum likelihood (ML) estimates of parameters or often fail convergence in cases that data is limited (e.g. limited number of subjects, few data per subject), parameters are highly nonnormally distributed, and/or complex model with many parameters ${ }^{2324)}$. In the cases, the MCMC Bayesian method which provides a large sample set of probable population parameters instead of providing point ML estimates, could be an option for parameter estimation, especially prior information can be utilized in general. The mean of the sample set are similar to exact maximum likelihood values with considerable stability, and the standard deviations of the samples are similar to standard errors obtained with maximum likelihood methods. In dasatinib development program in Japan, noncompartmental PK analysis was performed in a phase $1 / 2$ clinical trial (Study CA180-031) in Japanese subjects with imatinibresistant or -intolerant chronic phase CML who received dasatinib 50, 70 or $90 \mathrm{mg} \mathrm{BID}^{1,11}$. However, sparse $\mathrm{PK}$ sample collections were conducted in subsequent studies (Study CA180-036 and -138) and no intensive PK data of Japanese subjects is available at $100 \mathrm{mg}$ QD which was approved as the starting dose for the treatment of imatinib-resistant or -intolerant chronic phase CML. In the present analysis, because of nonconvergence in FOCE method, we employed BAYES method in combination with ITS method using integrated data across Japanese clinical trials to characterize PK profiles in Japanese patient population, and provide key PK parameters for the efficacy and the safety at tested dose including 100 
mg QD, which enable us to compare with PK data in non-Japanese.

\section{Materials and Methods}

\section{Data}

The Japanese PPK model of dasatinib was developed with 706 plasma concentration observations from 63 Japanese $\mathrm{CML}$ and $\mathrm{Ph}^{+}$ALL subjects who were resistant or intolerant to imatinib. These patients were enrolled in two phase $1 / 2$ clinical studies (Study CA180-031 and Study CA180-036) and a phase 2 clinical study (Study CA180-138) that were conducted to evaluate the efficacy, safety, and PK of dasatinib. The outcomes of these studies were previously reported ${ }^{1,11,12)}$.

Study CA180-031 was an open-label phase 1/2 study in which patients with $\mathrm{Ph}^{+}$chronic, accelerated or blast phase CML, primary or acquired resistance to imatinib, and with $\mathrm{Ph}^{+} \mathrm{ALL}$ resistant to past therapy were treated with 50, 70 or $90 \mathrm{mg}$ BID of dasatinib. Study CA180-036 was a phase $1 / 2$ study to document the long-term safety and efficacy of dasatinib at 50, 70 or $90 \mathrm{mg}$ BID in subjects with $\mathrm{Ph}^{+}$chronic, accelerated or blast phase CML, primary or acquired resistance to imatinib and with $\mathrm{Ph}^{+}$ALL resistant to past therapy, who completed the Study CA180-031. Study CA180-138 was a multi-center, open label, randomized, non-comparative phase 2 study to evaluate the efficacy at $100 \mathrm{mg}$ QD or $50 \mathrm{mg}$ BID in subjects with imatinib-resistant or -intolerant $\mathrm{Ph}^{+}$chronic phase CML. Study protocols and patient informed consents in these studies were approved by an institutional review board at each study center and conducted in accordance with the principles of the declaration of Helsinki and good clinical practice.

Intensive PK sampling at $0.5,1.0,1.5,2,3,4,5,6$, $8,12 \mathrm{hr}$ post-dose was performed on Day 1 and Day 28 in phase 1 portion of Study CA180-031. Six PK samples at any 3 or more visits of later than 7 days were preferably collected at pretreatment trough (within 1 hour prior to dosing) or any time between 3 and 10 hours following treatment in phase 1 extension portion and phase 2 portion in Study CA180-031, and in Study CA180-036 and Study CA180-138. The sampling scheme was chosen to be informative on $\mathrm{PK}$ parameter estimates, and on inter-occasion variability $^{25,26)}$.

The database that was used for PPK analysis in Japanese subjects consisted of 706 observations from
Table 1 Summary of baseline demographic and laboratory covariates included in the analysis

\begin{tabular}{ll}
\hline \multicolumn{1}{c}{ Covariate } & \multicolumn{1}{c}{ Median (Range) or Count (\%) } \\
\hline Age, year & $54(26-74)$, \\
& $<65: 53(84.1 \%)$, \\
& $65 \geq: 10(15.9 \%)$ \\
Weight, kg & $62(37-90)$ \\
BSA, m ${ }^{2}$ & $1.69(1.29-2.08)$ \\
BMI, kg/m ${ }^{2}$ & $23.0(14.3-32.8)$ \\
AST, U/L & $20(9-65)$ \\
ALT, U/L & $16(7-101)$ \\
Creatinine clearance ${ }^{\mathrm{a}}$, & $94.4(43.0-150)$ \\
mL/min & $6.6(0.9-89.2)$ \\
WBC, $\times 10^{3} \mathrm{c} / \mu \mathrm{L}$ & $11.9(6.5-16.2)$ \\
Hemoglobin, g/dL, & Chronic phase CML : 53 (84.1\%) \\
time variant & Accelerated phase CML : $5(7.9 \%)$ \\
Disease Status & Blast phase CML : $1 \quad(1.6 \%)$ \\
& Ph ${ }^{+}$ALL : 4 (6.4\%) \\
\hline
\end{tabular}

ALT : alanine aminotransferase, AST : aspartate aminotransferase, BMI : body mass index, BSA : body surface area, CML : chronic myeloid leukemia, $\mathrm{Ph}^{+} \mathrm{ALL}$ : Philadelphia chromosomepositive acute lymphoblastic leukemia, WBC : white blood cells.

${ }^{a}:$ Calculated creatinine clearance values were capped at 150 $\mathrm{mL} / \mathrm{min}$ for the purpose of covariate modeling to ensure physiological plausibility.

a total of 63 subjects. Summary of the baseline demographic and clinical laboratory covariates for these subjects is given in Table 1.

\section{Assay of Plasma Dasatinib Concentrations}

Plasma concentrations of dasatinib were measured by a validated liquid chromatography tandem mass spectrometry (LC/MS/MS) method with a calibration curve range of $1 \mathrm{ng} / \mathrm{mL}$ to $1000 \mathrm{ng} / \mathrm{mL}^{21)}$. All values below the lower limit of quantification ( $1 \mathrm{ng} /$ $\mathrm{mL}$ ) were excluded from this PPK analysis. The between-run and within-run precision for analytical quality control samples were no greater than $7.8 \%$ and $4.2 \%$ coefficient of variation (CV), respectively, with deviations from the nominal concentrations of no more than $\pm 6.3 \%$, indicating that the bioanalytical method performance was precise and accurate for the analysis of dasatinib in human plasma in all of three Japanese clinical studies.

\section{Population PK Model Development}

Plasma concentration-time data from Japanese subjects were analyzed using a non-linear mixed effects modeling approach, as implemented in the NONMEM 7 computer program (ICON Develop- 
ment Solutions, MD, USA). Based on previous PPK analyses and the graphical assessment of concentration data from Japanese subjects, the data was fitted to a two-compartment pharmacokinetic model with first-order absorption and elimination which was parameterized with absorption rate constant (KA), apparent clearance $(\mathrm{CL} / \mathrm{F})$, apparent volume of central compartment $(\mathrm{Vc} / \mathrm{F})$, apparent intercompartmental clearance $(\mathrm{Q} / \mathrm{F})$ and apparent volume of peripheral compartment $(\mathrm{Vp} / \mathrm{F})$ as fixed effect parameters. Inter-occasion variability (IOV) on relative bioavailability $\left(\mathrm{F}_{\mathrm{R}}\right)$ and inter-individual variabilities (IIV) on KA, CL/F, Vc/F, Q/F, Vp/F or $F_{R}$ were also estimated as random effect parameters.

In previous PPK analysis in non-Japanese subjects, FOCE with interaction was utilized for parameter estimation. However, because of the limited data compared to the non-Japanese data and nonconvergence in FOCE method, a stable estimation method, BAYES methods, were applied for this PPK analysis. The analysis was initially done by ITS method and then, the result was used as the initial values for the subsequent full MCMC Bayesian analysis by BAYES method which could provide less bias than linearization maximization methods such as FOCE and ITS $^{23}$. The prior information in this analysis were coded for fixed and random effect parameters to be highly uninformative, and 3000 iterations were set for NITER option to construct the stationary distribution in the BAYES analysis. Convergence of ITS and BAYES methods was assessed by linear regression on all of the population parameters versus iteration (CTYPE $=3$ ), and convergence or stationary distribution was deemed to have been achieved when none of the slopes were significant $(\mathrm{p}<0.05)^{23)}$. To finally confirm if the Markov chain has reached its stationary posterior distribution in BAYES method, trace plots of iteration versus parameter estimates and Geweke convergence diagnostic test statistic were also used $^{27-29)}$. This statistic is a widely accepted convergence diagnostic in MCMC analysis, which is based on an assessment of the equality of parameter means from the early and latter part of the post-burn-in iterations (the first $10 \%$ and the last $50 \%$ ) using the $z$-statistic. If Geweke convergence diagnostic test statistic (Z-score) of a parameter is more than 1.0 , then the distribution of post-burn-in iterations was not deemed to be stationary ${ }^{27,29)}$. In the case, the larger number of iteration was set for NBURN option without the CTYPE option in BAYES method to observe the longer burn-in phase.

The IIV and IOV were described by an exponential model and estimated by Gibbs sampling method ${ }^{23)}$. The residual error was described by additive lognormal model and estimated by MetropolisHastings sampling method.

\section{Covariate Selection}

The baseline background (body size including body weight, BMI and BSA, age, gender, disease status [DS], prior imatinib treatment, nominal dose), baseline hepatic status (alanine aminotransferase [ALT] and aspartate aminotransferase [AST]), baseline renal status (creatinine clearance calculated by the Cockcroft-Gault equation ${ }^{30}$ [BCrCL]), and timevarying clinical laboratory covariates (hemoglobin and white blood cell count) were included in the population $\mathrm{PK}$ analysis dataset.

Continuous covariates were modeled using a general slope function :

$$
\begin{aligned}
& M U \_i=\log P_{\text {avg }}+\theta \times \log (\text { COV } i) \\
& P i=\exp \left(M U_{-} i+\eta_{p i}\right)
\end{aligned}
$$

For each parameter $i$ that has an $\eta$ associated with it, and MU_i is a function of a population mean PK parameter $\left(\mathrm{P}_{\text {avg }}\right)$. COVi represents individual covariate values normalized by the approximate median value for the subject population, and $\theta$ represents a scale factor. $\mathrm{P}_{\mathrm{i}}$ is the value of parameter $\mathrm{P}$ for the $\mathrm{i}^{\text {th }}$ individual, and $\eta_{\mathrm{pi}}$ is a realization of a normally distributed random variable with zero mean and variance $\omega_{P}^{2}$. Categorical covariates were modeled as follows ;

$$
\begin{aligned}
& \left.M U_{\_} i=\log P_{\text {avg } 1} \quad \text { (If covariate }=1\right) \\
& M U_{\_} i=\log P_{\text {avg } 2} \quad(\text { If covariate }=2) \\
& P i=\exp \left(M U_{-} i+\eta_{p i}\right)
\end{aligned}
$$

In the first step, the covariate-parameter relationships were visually assessed by the plots of individual parameters vs individual covariate values for the selection of possible covariates. Once possible covariates were initially selected, then covariate models were developed by a forward inclusion method in the second step. Deviance information criteria (DIC) was employed for the covariate selection ${ }^{31-33)}$. The model with a smaller DIC value is usually considered more parsimonious than the one with a large DIC values. Covariates would be considered to be clinically 
relevant and remained in the model if DIC indicated that a covariate model was better fit to the data, and the covariate provided more than $\pm 20 \%$ change relative to the reference value in a PK parameter.

Because $\mathrm{C}_{\text {avg ss }}$ and $\mathrm{C}_{\text {min ss }}$ are key parameters for the efficacy and the safety, respectively, the covariate selection for $\mathrm{CL} / \mathrm{F}$ was especially focused on in this analysis. Baseline creatinine clearance (BCrCL), gender, disease status (DS), and age were tested as covariates on $\mathrm{CL} / \mathrm{F}$. Age was categorized as $<65$ years and $\geq 65$ years to provide the supportive information of dasatinib exposure for elderly population.

\section{PK Linearity and Food Effect}

Food effect (administration of dasatinib with/without food within 30 min before or after administration) was tested on $F_{R}$ by the categorical covariate model (Equation 2) and the impact was estimated since it was not assessed in previous PPK analyses and no PK data after administration of dasatinib with food consumption is available in Japanese population. Exploratory assessment of PK linearity in Japanese subjects with chronic phase CML was performed in Study CA180-031. However, no meaningful conclusion could be drawn due to the limited number of subjects. In this PPK analysis, the dose effect on CL/F was also tested using the general slope function (Equation 1) to assess PK linearity in Japanese subjects, which would provide supportive and more robust information in terms of PK linearity in Japanese population based on the integrated PK data across Japanese clinical trials.

\section{Model Evaluation and Application}

Diagnostic plots were used to assess the goodnessof-fit of this model, and to help model evaluation. The stability of the parameters on a selected model was assessed using 95\% credible intervals (CIs) of each parameters calculated from the stationary phase of the MCMC. For predictive performance check, simulation was conducted for each 500 simulations at each dosing regimens. Population mean and the 95\% prediction intervals of the simulated concentrationtime profiles were constructed. The observed data was overlaid on the same plot for a visual check. In model application, the steady-state exposure $\left(\mathrm{C}_{\min s \mathrm{~s}}\right.$, $\mathrm{C}_{\max s s}, \mathrm{AUC}_{\mathrm{ss}}$ and $\mathrm{C}_{\text {avg ss }}$ ) of all subjects in the PPK analysis were predicted from the final PPK model, and summarized to compare dasatinib PK in the Japanese CML patients with that in the global CML patient population.

\section{Results}

Observed dasatinib concentration-time profiles were biphasic with a rapid distribution phase and a slower elimination phase and therefore, the dasatinib PK data in Japanese subjects were initially described by a linear two-compartment PPK model with IOV on $F_{R}$, which is the identical to the structural model in non-Japanese subjects ${ }^{22)}$.

The result of covariate selection is provided below, followed by summary of model evaluation and application of the final model including key PK parameters of subjects with chronic phase CML who administered at $100 \mathrm{mg}$ QD in Study CA180-138.

\section{Covariate Selection}

Covariates were screened by the visual assessment of covariate-parameter plots (Fig. 1), and BCrCL, gender, DS and age were initially selected as possible covariates on $\mathrm{CL} / \mathrm{F}$ for the following reasons : 1) there is a trend towards slightly increasing $\mathrm{CL} / \mathrm{F}$ with increasing $\mathrm{BCrCL} ; 2)$ median $\mathrm{CL} / \mathrm{F}$ of female subjects $(216 \mathrm{~L} / \mathrm{h})$ was higher than that of male subjects (187 L/h) ; 3) median CL/F of subjects with chronic phase CML $(202 \mathrm{~L} / \mathrm{h})$ was smaller than that of subjects with advanced phase $(230 \mathrm{~L} / \mathrm{h}) ; 4)$ median $\mathrm{CL} / \mathrm{F}$ of elderly subjects $(181 \mathrm{~L} / \mathrm{h})$ was smaller than that of young subjects $(208 \mathrm{~L} / \mathrm{h})$. Each of the covariate effects were tested by modeling and evaluated by DIC. Other examined covariates didn't show any trend by visual assessment, and therefore, were not included forward.

For the covariates of $\mathrm{BCrCL}$, gender and DS, DIC of covariate models were higher than that of the PPK model without the covariate (Table 2), suggesting that the effect of $\mathrm{BCrCL}$, gender and DS had no significant impact on $\mathrm{CL} / \mathrm{F}$. Addition of the effect of age to the PPK model lowered DIC by 9 . However, median values of population mean $\mathrm{CL} / \mathrm{F}$ from 3000 iterations in young ( $\leq 65$ years) and elderly $(>65$ years) subjects indicated dasatinib $\mathrm{CL} / \mathrm{F}$ decreased by $9 \%$ for elderly subjects, compared with young subjects. These results suggested that the effect of age on $\mathrm{CL} / \mathrm{F}$ is not clinically relevant, and therefore, age wasn't eventually selected as a covariate. 

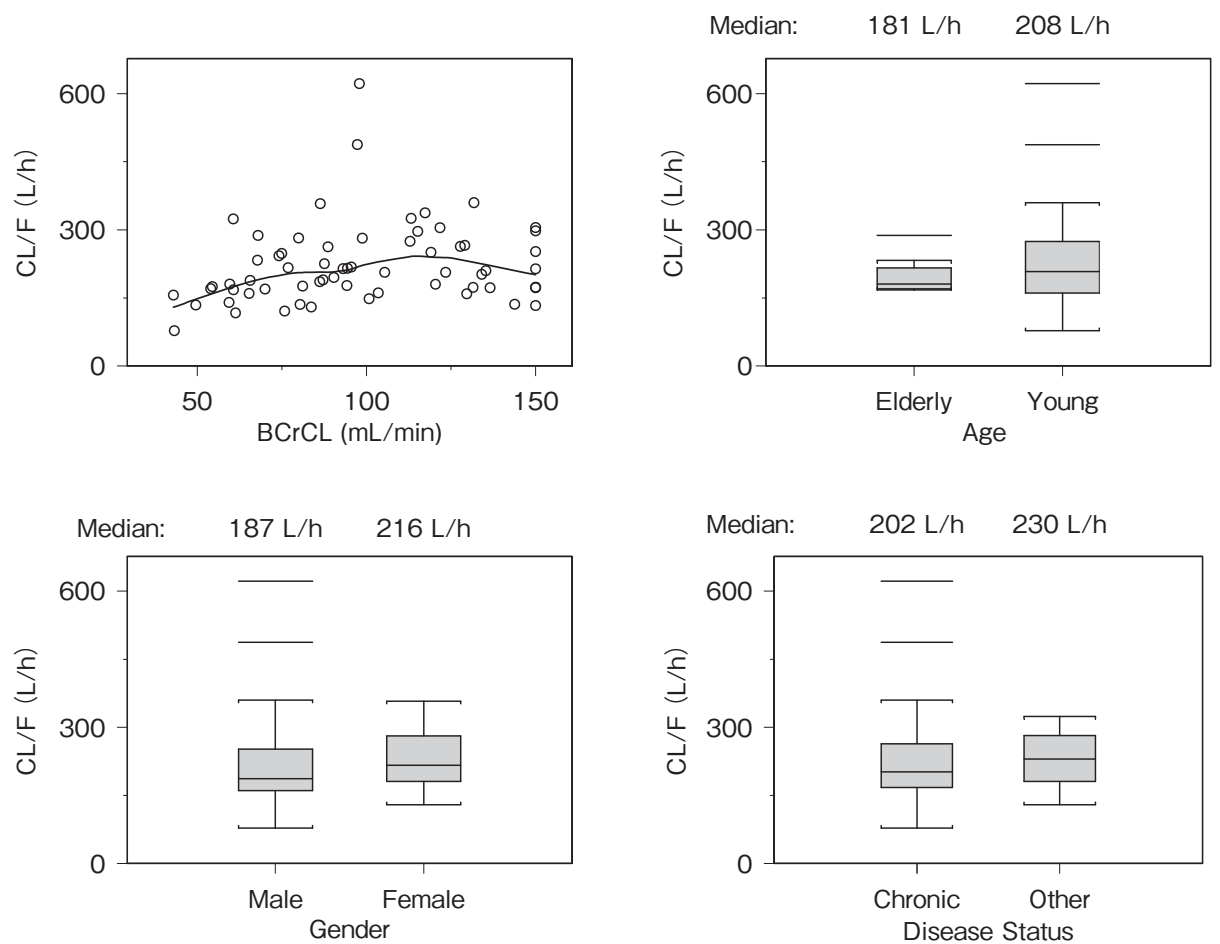

Fig. 1 The relationship between individual $\mathrm{CL} / \mathrm{F}$ and the covariates (BCrCL, age, gender and DS) in Japanese subjects

The solid line on the upper left figure is a Loess smooth. BCrCL represents baseline creatinine clearance. Age was categorized as $<65$ years (Young, $\mathrm{n}=53$ ) and $\geq 65$ years (Elderly, $\mathrm{n}=10$ ).

The number of male and female are 42 and 21 subjects, respectively.

Disease status on the bottom right figure was categorized chronic phase CML (Chronic, $\mathrm{n}=53$ ) and other $(\mathrm{n}=10)$ including accelerated and blast phase $\mathrm{CML}$, and $\mathrm{Ph}^{+} \mathrm{ALL}$.

Table 2 Difference of DIC between PPK models with and without the effect of covariate in covariate selection

\begin{tabular}{lcr}
\hline \multicolumn{1}{c}{ Covariate } & Parameter $^{\mathrm{a}}$ & $\Delta \mathrm{DIC}^{\mathrm{b}}$ \\
\hline Baseline Creatinine Clearance & $\mathrm{CL} / \mathrm{F}$ & 31.0 \\
Disease Status & $\mathrm{CL} / \mathrm{F}$ & 56.7 \\
Gender & $\mathrm{CL} / \mathrm{F}$ & 9.9 \\
Age & $\mathrm{CL} / \mathrm{F}$ & -9.3 \\
Dose & $\mathrm{CL} / \mathrm{F}$ & 38.4 \\
Food & $\mathrm{F}_{\mathrm{R}}$ & 40.3 \\
\hline
\end{tabular}

${ }^{\mathrm{a}}$ : Parameter to which the covariate effect was added.

b : Difference of DIC from PPK model without the effect of covariate.

\section{PK Linearity and Food Effect}

No significant dose effect was found on CL/F. The PPK model with the effect of the dose on $\mathrm{CL} / \mathrm{F}$ increased the DIC value by 36 , and $95 \%$ CI of the dose effect ( -0.02 to 0.67 ) included zero, indicating that the linear PPK model without the dose effect is better fit to the data and dasatinib PK does not deviate from dose linearity at tested dose range in Japanese subjects. No apparent food effect was also identified on $\mathrm{F}_{\mathrm{R}}$. The model with food effect increased DIC by 40 , and the $95 \%$ CI of food effect (ratio of without/ with food within $30 \mathrm{~min}$ before or after administration) was $0.85^{-1.20 .}$

\section{Final Model Evaluation and Application}

None of the parameter-covariate relationships was retained in the linear two-compartment PPK model due to lack of either sufficient difference in DIC, or clinical relevance. Representative trace plots and all of the model parameter estimates are presented in Figure 2 and Table 3, respectively. Trace plots and Geweke convergence diagnostic test statistics ranging from 0.05 to 0.992 demonstrated the achievement of stationary distribution on each of model parameters in the final model. Diagnostic plots of goodness-of-fit for the final model also showed there was reasonable agreement between a wide range of observed concentrations and the corresponding typical and individual predicted values (Fig. 3). The diagnostic plot of the weighted residual versus time also indicates no evidence of autoinhibition or 

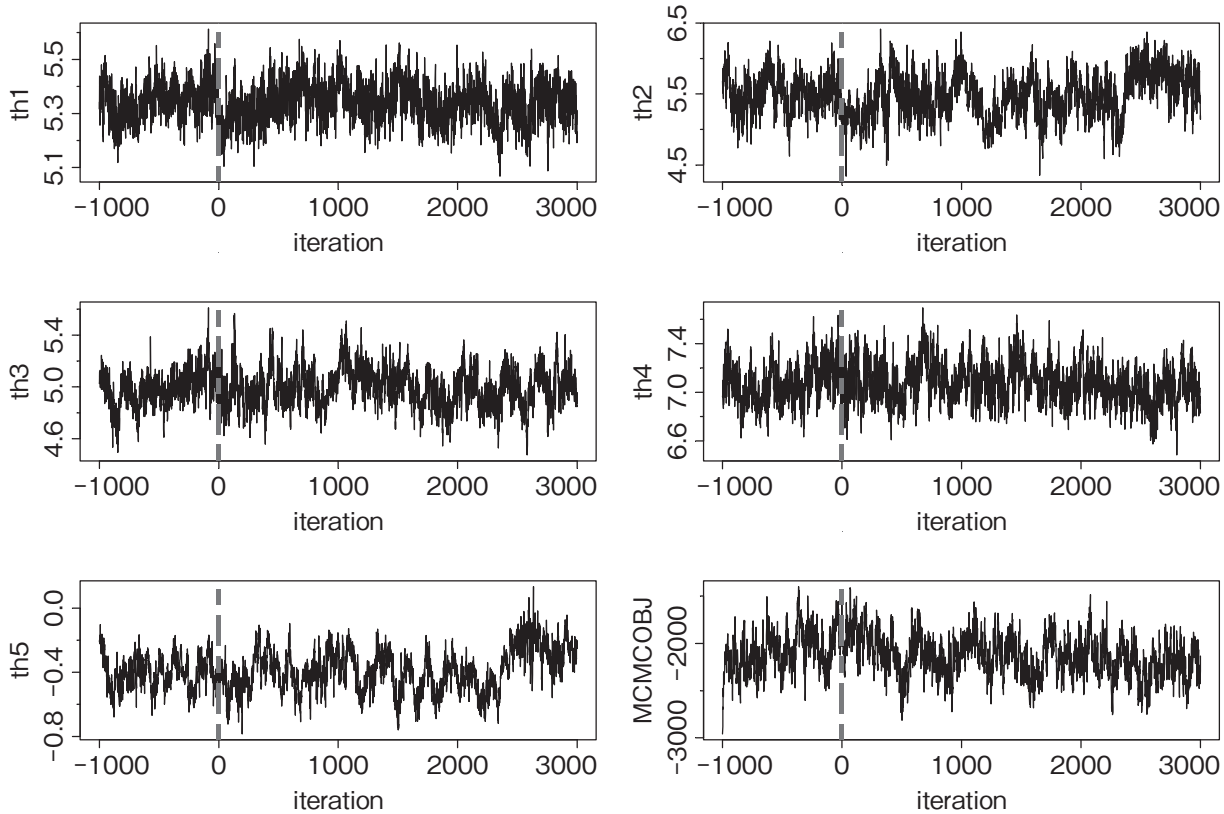

Fig. 2 Representative trace plots of PK parameters and objective function values generated from MCMC algorithm in final PPK model

The part of iteration less than zero is in burn-in phase decided by the termination test option. th1, th2, th3, th4, th5 and MCMCOBJ represent CL/F, Vc/F, Q/F, Vp/F, KA and objective function value, respectively. Trace plots of the other parameters also showed no marked trend and Geweke convergence diagnostic test statistics of all of model parameters listed in Table 3 ranged from 0.05 to 0.992 , demonstrating the achievement of stationary distribution.

Table 3 Parameter estimates of final PPK model

\begin{tabular}{|c|c|c|c|}
\hline Parameter & Estimate $^{\mathrm{a}}$ & Lower limit of $95 \% \mathrm{CI}^{\mathrm{b}}$ & Upper limit of $95 \% \mathrm{CI}^{\mathrm{b}}$ \\
\hline \multicolumn{4}{|l|}{ Fixed-Effects } \\
\hline $\mathrm{CL} / \mathrm{F}[\mathrm{L} / \mathrm{h}]$ & 208 & 180 & 240 \\
\hline $\mathrm{Vc} / \mathrm{F}[\mathrm{L}]$ & 239 & 132 & 426 \\
\hline $\mathrm{Q} / \mathrm{F}[\mathrm{L} / \mathrm{h}]$ & 148 & 111 & 201 \\
\hline $\mathrm{Vp} / \mathrm{F}[\mathrm{L}]$ & 1177 & 876 & 1654 \\
\hline $\mathrm{KA}[1 / \mathrm{h}]$ & 0.680 & 0.531 & 0.877 \\
\hline \multicolumn{4}{|l|}{ Random-Effects } \\
\hline$\omega_{\mathrm{CL} / \mathrm{F}}^{2}$ & $0.1755(42 \%)$ & 0.1022 & 0.2852 \\
\hline$\omega_{\mathrm{Vc} / \mathrm{F}}^{2}$ & $1.6037(127 \%)$ & 0.5604 & 3.3211 \\
\hline$\omega_{\mathrm{Q} / \mathrm{F}}^{2}$ & $0.3893(62 \%)$ & 0.1686 & 0.7548 \\
\hline$\omega_{\mathrm{Vp} / \mathrm{F}}^{2}$ & $0.6310(79 \%)$ & 0.2858 & 1.1610 \\
\hline$\omega_{\mathrm{KA}}^{2}$ & $0.1340(37 \%)$ & 0.0441 & 0.3526 \\
\hline$\omega_{\mathrm{FR}}^{2}$ & $0.0819(29 \%)$ & 0.0363 & 0.1581 \\
\hline$\omega_{\mathrm{FR} \text {, iov }}^{2}$ & $0.1762(42 \%)$ & 0.0932 & 0.3015 \\
\hline$\omega_{\mathrm{CL} / \mathrm{F}}^{2}: \mathrm{Vc} / \mathrm{F}$ & $0.3403(0.641)$ & 0.0977 & 0.6783 \\
\hline$\omega_{\mathrm{CL} / \mathrm{F}: \mathrm{Q} / \mathrm{F}}^{2}$ & $0.1593(0.609)$ & 0.0513 & 0.3137 \\
\hline$\omega_{\mathrm{CL} / \mathrm{F}}^{2}: \mathrm{Vp} / \mathrm{F}$ & $0.2234(0.671)$ & 0.0838 & 0.4083 \\
\hline$\omega_{\mathrm{Vc} / \mathrm{F}: \mathrm{Q} / \mathrm{F}}^{2}$ & $0.3888(0.492)$ & 0.0019 & 0.9021 \\
\hline$\omega_{\mathrm{Vc} / \mathrm{F}}^{2}: \mathrm{Vp} / \mathrm{F}$ & $0.5382(0.535)$ & 0.0788 & 1.1569 \\
\hline$\omega_{\mathrm{Q} / \mathrm{F}: \mathrm{Vp} / \mathrm{F}}^{2}$ & $0.3642(0.735)$ & 0.1337 & 0.7251 \\
\hline \multicolumn{4}{|c|}{ Residual Error (Log) } \\
\hline$\theta_{\mathrm{LOG}}$ & 0.522 & 0.501 & 0.542 \\
\hline
\end{tabular}



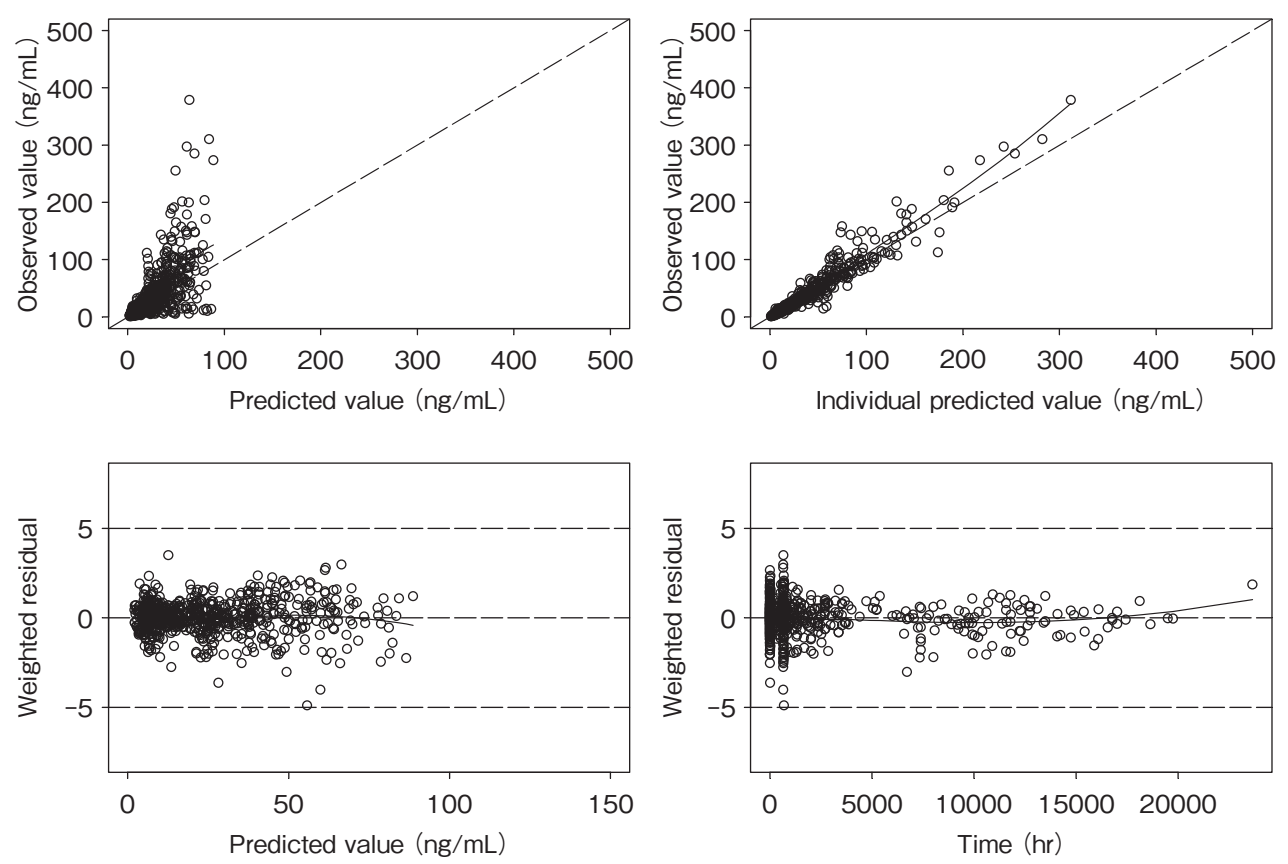

Fig. 3 Basic goodness-of-fit diagnostic plots for final PPK model

Monte-Carlo generated predicted concentration versus observed dasatinib concentration, individual fitted concentration versus observed dasatinib concentration, Monte-Carlo generated weighted residual versus the predicted concentration, and the weighted residuals versus time. Solid lines are loess smooth lines.

autoinduction of elimination. The visual predictive check results for data at $100 \mathrm{mg}$ QD and $70 \mathrm{mg}$ BID which were approved in Japan as dosing regimens for the treatment of chronic and advanced phase CML, respectively, also indicated that the model described the overall concentration time-profile reasonably well (Fig. 4). The advantage of introducing IOV on $F_{R}$ is presented in Figure 5. The subject shown in Figure 5 had the highest variability of the dasatinib exposure between visits. Although $\mathrm{C}_{\max }$ and AUC on Day 28 decreased by $90 \%$ and $73 \%$, respectively, compared to those on Day 1, plasma concentration-time profiles were well estimated on each days, indicating the ability of the PPK model to describe different concentration-time profiles for the same subject on different days. For this subject, the information of concomitant medication such as gastric pH modulators, strong CYP3A4 inhibitors, or strong CYP3A4 inducers which have the potential to decrease dasatinib plasma concentrations, was not available. The exact reason for the large observed betweenvisit variability is therefore, unknown ${ }^{1}$. Similarly, between-visit difference in dasatinib exposure was reported in the PPK analysis in non-Japanese subjects $^{22)}$.
The steady-state exposure $\left(\mathrm{C}_{\min s \mathrm{~s}}, \mathrm{C}_{\max s \mathrm{ss}}, \mathrm{AUC}_{\mathrm{ss}}\right.$, and $\mathrm{C}_{\text {avg ss }}$ ) for all subjects in the PPK analysis were predicted from the final PPK model and summarized in Table 4. PK parameter estimates of Japanese subjects at $100 \mathrm{mg}$ QD and $70 \mathrm{mg}$ BID in this analysis was compared with the results in previous PPK analysis in non-Japanese subjects by boxplots given in Figure $6^{22}$. Although $\mathrm{C}_{\text {min ss }}$ values of subjects with chronic phase CML seem to be scattered in the higher regions of over the median $\mathrm{C}_{\min }$ ss of the non-Japanese subjects, the magnitude of the difference was small. Median $\mathrm{C}_{\text {min ss }}$ at $100 \mathrm{mg}$ QD were 3.3 and $1.8 \mathrm{ng} / \mathrm{mL}$ in the Japanese and non-Japanese subjects, respectively. Given the sample size and the extend of the steady-state exposure variability, the distribution of the other individual parameter estimates in Japanese $\mathrm{CML}$ and $\mathrm{Ph}^{+} \mathrm{ALL}$ subjects was generally considered to overlap with the non-Japanese subjects and no marked difference was observed between two populations.

\section{Discussion}

Population PK of dasatinib in Japanese subjects with $\mathrm{CML}$ and $\mathrm{Ph}^{+}$ALL was characterized and dasatinib exposure at various doses including $100 \mathrm{mg}$ 

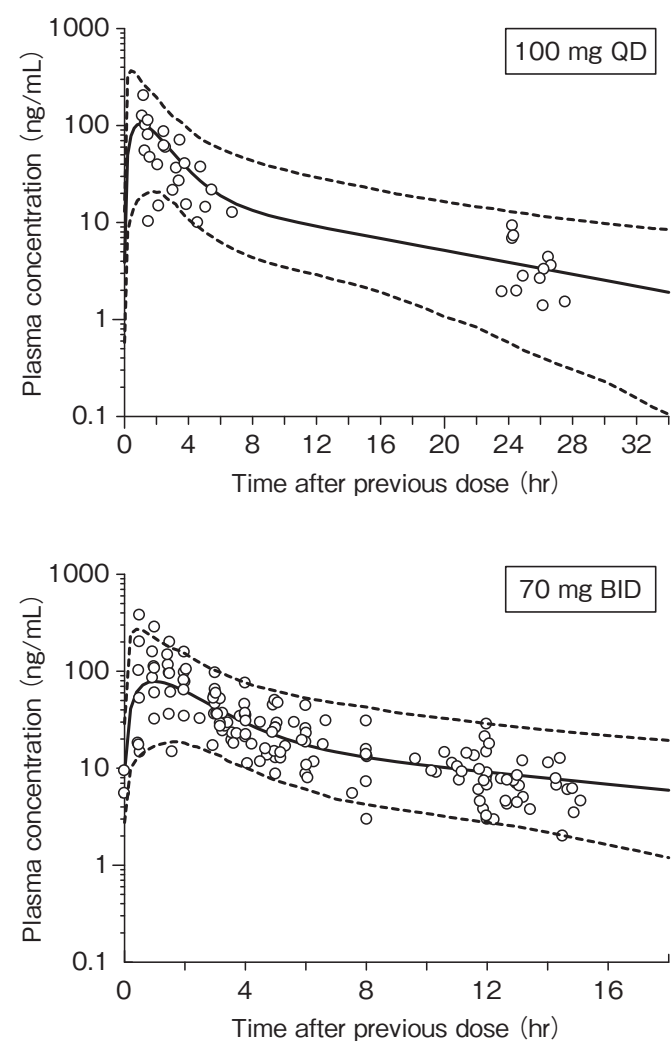

Fig. 4 Observed dasatinib concentrations and 95\% prediction intervals at steady-state in final model

Dash lines represent lower and upper limit of 95\% prediction intervals and center solid lines indicate population mean plasma dasatinib concentrations. Open circles show observed dasatinib concentrations.

QD were compared with that in non-Japanese subjects with CML and $\mathrm{Ph}^{+} \mathrm{ALL}$ in order to interpret the Japanese data in the context of the larger global clinical database.

It has been demonstrated that for PPK analysis, linearization maximization methods such as FOCE could introduce bias into parameter estimates, especially when the assumption of normal parameter distributions does not hold. Dasatinib PK has shown moderate to high variability (including IIV and IOV). In the current analysis, it is not unexpected to see that FOCE method failed to converge, and the shrink values of ETAs for $\mathrm{KA}$ and $\mathrm{F}_{\mathrm{R}}$ were greater than $90 \%$ in ITS method, suggesting that those parameters are non-normally distributed. The typical values of KA and $F_{R}$ were not estimated precisely as indicated by the large standard errors. Savic and Karlsson proposed when shrinkage is high in FOCE method, more direct population model estimation needs to be employed in model building and evaluation ${ }^{34)}$. Full

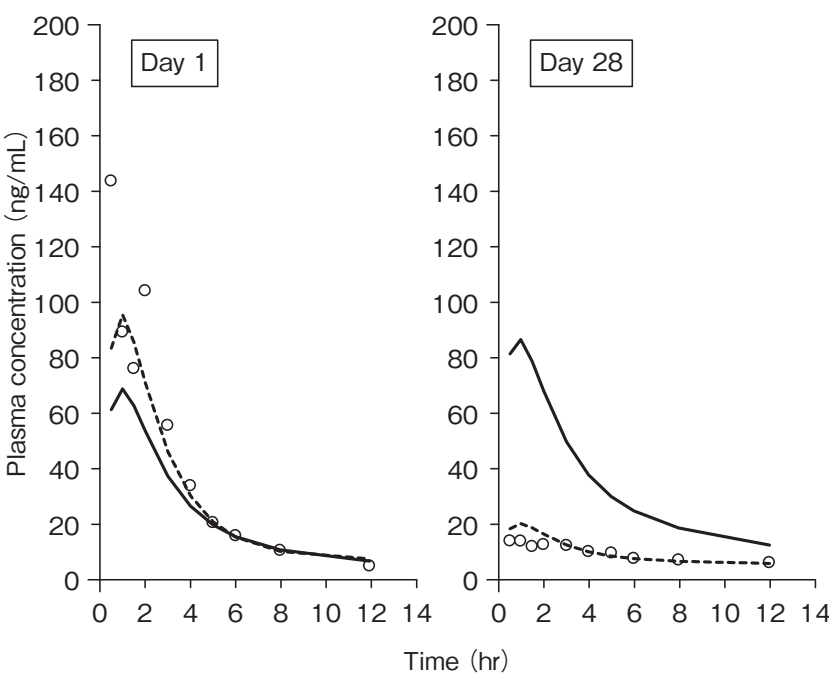

Fig. 5 Observed and predicted dasatinib concentrationtime profiles in a representative subject receiving $90 \mathrm{mg}$ BID (Day 1 and Day 28) in Study CA180031

Dash lines represent individual predicted dasatinib concentrations and solid lines indicate population mean plasma dasatinib concentrations. Open circles show observed dasatinib concentrations.

Bayesian approach implemented by MCMC method on the other hand, does not make assumption on the form of parameter distributions, and it draws samples from a proposal distribution and then directly obtains the stationary posterior distribution to be used for statistical summaries. It is therefore, adopted in the current analysis as the preferred estimate methods. It turned out that this approach is more stable and remarkably improved the precision of ETAs for KA and $\mathrm{F}_{\mathrm{R}}$ where their relative standard errors were $61 \%$ and $39 \%$, respectively. Such stability and improvement would be beneficial to subsequent model building including covariate selections. In any practical application of MCMC, there are important decisions to be made, including the number of initial iterations discarded as the burn-in phase and the assessment of convergence after the selected burn-in phase. To support these decisions, we employed the option of termination test (CTYPE) as implemented in NONMEM 7, trace plots and Geweke convergence diagnostic test in this analysis. CTYPE option is convenient to decide the burn-in phase for estimating the model parameters, but the decided burn-in phase might not be robust to compute appropriate estimates. In several problems, MCMC object function of the stationary distribution did not denote all of the model parameters also reached the stationary distri- 
Table 4 Mean $(\mathrm{CV} \%)$ of simulated steady-state exposure in Japanese subjects

\begin{tabular}{|c|c|c|c|c|c|c|}
\hline $\begin{array}{l}\text { Disease } \\
\text { State }\end{array}$ & $\begin{array}{l}\text { Dosing } \\
\text { regimen }\end{array}$ & $\mathrm{N}^{\mathrm{a}}$ & $\begin{array}{c}\mathrm{C}_{\max \mathrm{ss}} \\
(\mathrm{ng} / \mathrm{mL})\end{array}$ & $\begin{array}{c}\mathrm{AUC}_{\mathrm{ss}} \\
(\mathrm{ng} \cdot \mathrm{h} / \mathrm{mL})\end{array}$ & $\begin{array}{c}\mathrm{C}_{\text {min ss }} \\
(\mathrm{ng} / \mathrm{mL})\end{array}$ & $\begin{array}{c}\mathrm{C}_{\text {avg ss }} \\
(\mathrm{ng} / \mathrm{mL})\end{array}$ \\
\hline Chronic & $100 \mathrm{mg}$ QD & 10 & $\begin{array}{c}81.57 \\
(87)\end{array}$ & $\begin{array}{c}426.29 \\
(45)\end{array}$ & $\begin{array}{l}3.64 \\
(39)\end{array}$ & $\begin{array}{c}17.76 \\
(45)\end{array}$ \\
\hline Chronic & $50 \mathrm{mg} \mathrm{BID}$ & 33 & $\begin{array}{c}65.67 \\
(41)\end{array}$ & $\begin{array}{l}281.49 \\
(33)\end{array}$ & $\begin{array}{l}7.81 \\
(39)\end{array}$ & $\begin{array}{c}23.46 \\
(33)\end{array}$ \\
\hline $\begin{array}{l}\text { Chronic \& } \\
\text { Advanced }^{\mathrm{b}}\end{array}$ & $70 \mathrm{mg}$ BID & 15 & $\begin{array}{c}77.87 \\
(46)\end{array}$ & $\begin{array}{c}311.81 \\
(30)\end{array}$ & $\begin{array}{l}8.26 \\
(38)\end{array}$ & $\begin{array}{c}25.98 \\
(30)\end{array}$ \\
\hline Chronic & $90 \mathrm{mg}$ BID & 4 & $\begin{array}{c}127.73 \\
(113)\end{array}$ & $\begin{array}{c}451.79 \\
(68)\end{array}$ & $\begin{array}{c}11.77 \\
(24)\end{array}$ & $\begin{array}{c}37.65 \\
(68)\end{array}$ \\
\hline
\end{tabular}

${ }^{\mathrm{a}}$ : One subject was not included in this summary because the dosage had been reduced from $100 \mathrm{mg}$ QD to $80 \mathrm{mg}$ QD when PK samples were collected.

${ }^{\mathrm{b}}$ : Nine, four and two subjects with chronic phase CML, accelerated phase CML and $\mathrm{Ph}^{+} \mathrm{ALL}$, respectively.

$\underline{100 \mathrm{mg} Q \mathrm{D}}$

Median: $\quad 84 \mathrm{ng} / \mathrm{mL} \quad 76 \mathrm{ng} / \mathrm{mL}$
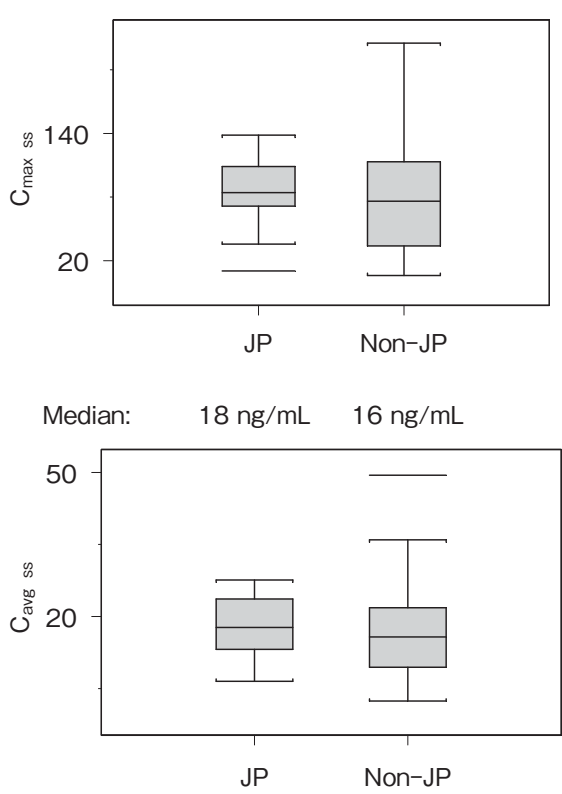

Median: $\quad 3.3 \mathrm{ng} / \mathrm{mL} \quad 1.8 \mathrm{ng} / \mathrm{mL}$

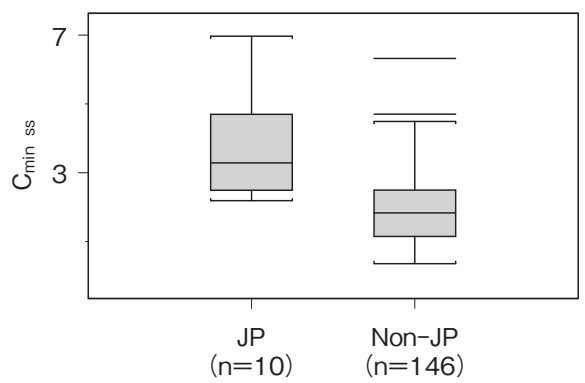

$70 \mathrm{mg}$ BID
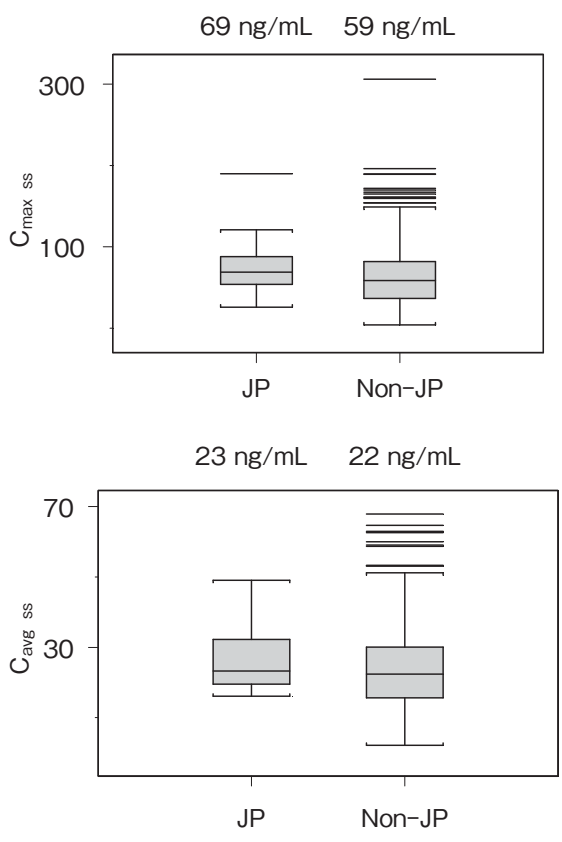

$7.7 \mathrm{ng} / \mathrm{mL} \quad 6.5 \mathrm{ng} / \mathrm{mL}$

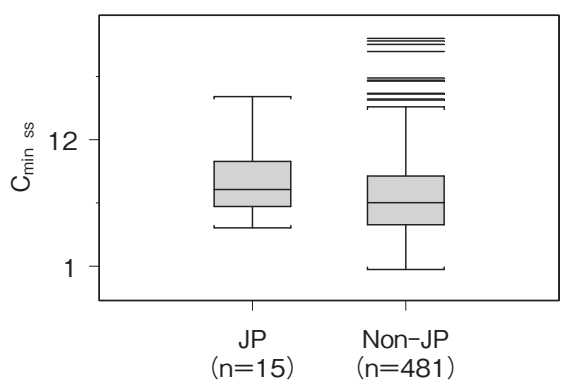

Fig. 6 Box-and -whisker plots of PK parameters at steady state for dasatinib at 100 $\mathrm{mg} \mathrm{QD}$ and $70 \mathrm{mg}$ BID in Japanese (JP) and non-Japanese (Non-JP) subjects with imatinib-resistant or -intolerant $\mathrm{CML}$ and $\mathrm{Ph}^{+} \mathrm{ALL}^{22}$ 
bution, and suggested that visual inspection and convergence diagnostics such as trace plots and Geweke's convergence diagnostic test statistics should be used in addition to CTYPE option by each of model parameters in BAYES method.

For covariate selection in FOCE method, loglikelihood ratio test (LRT) or AIC is commonly utilized. BAYES method doesn't provide a maximum likelihood objective function for LRT and AIC, but, DIC which is intended as a generalization of AIC and preferably used in model selection when the posterior distributions of the model parameters have been obtained by MCMC simulation can be calculated ${ }^{31-33)}$. The PPK model with the age effect on $\mathrm{CL} / \mathrm{F}$ lowered DIC by 9 compared with the PPK model without the age effect, suggesting the improvement of the fit to the data by adding the age effect. However, the impact of age on $\mathrm{CL} / \mathrm{F}$ indicated the age effect on $\mathrm{CL} /$ $\mathrm{F}$ is not clinically relevant. Eventually, none of the covariate was retained in the PPK model which is identical to the latest model in non-Japanese subjects $^{22)}$. This population PK analysis demonstrated that no dose adjustment in terms of the exposure is warranted for body size, age, or gender, in Japanese patients. Also, the pharmacokinetic parameters for dasatinib are not dependent on hepatic or renal status within the limited range of baseline renal and hepatic clinical laboratory parameters observed in Japanese subjects.

In the present analysis, the IOV on $F_{R}$, which was included in the PPK model to account for the variable plasma concentration-time profiles among days in a same subject, was $42 \%$ (Table 3 ). The reasons for the observed in dasatinib PK are unknown, however, the possible major factors for high variability are change of CYP3A4 activity and/or gastric $\mathrm{pH}^{1,20)}$. No assessment of CYP3A4 activity was conducted in Study CA180-031, -036 and -138 but the inter-visit variability in CYP3A4 activity including concomitant medications (such as CYP3A4 inhibitors, or inducers) are likely to play an important role in the IOV since their impact on the dasatinib exposure has been demonstrated in clinical pharmacology studies. Dasatinib PK also depends on gastric $\mathrm{pH}$ since the drug's solubility decreases as $\mathrm{pH}$ increases. The drug-drug interaction study with Maalox (aluminum hydroxide/magnesium hydroxide-containing antacid) and famotidine suggested that the absorption of dasatinib is significantly changed under the gastric environment, and that the inter-visit variability in gastric absorption might lead to the IOV in dasatinib PK. However, it is unlikely that the inter-visit variability in gastric $\mathrm{pH}$ or CYP3A4 activity by concomitant medications mainly caused the IOV in the present analysis because CYP3A4/5 inducers and inhibitors were not given concomitantly around days for PK sampling collections in Study CA180-031, -036 and -138, and only a subject received famotidine concomitantly on Day 1 in Study CA180-031. Gastric absorption may also be variable due to gastric $\mathrm{pH}$ variations caused by diet. Modeling approach enables us to assess the impact of food effect by taking into account the IOV. No apparent food effect was found in this analysis, and adding the food effect to the final PPK model didn't result in reduction of IOV, suggesting that IOV is not mainly explained by the occasions of dosing with/ without food consumption. In theory, other factors including subject compliance, disease severity, and drug absorption/elimination organ status, may contribute to the IOV.

Initially, $50 \mathrm{mg}$ BID was proposed for the treatment to imatinib-resistant or -intolerant chronic phase CML patient in initial new drug application (NDA) for dasatinib in Japan in 2007, but $100 \mathrm{mg}$ QD was finally approved based on the risk-benefit assessment between $50 \mathrm{mg}$ BID and $100 \mathrm{mg}$ QD in phase 3 Study CA180-034 ${ }^{13)}$, the E-R analysis ${ }^{22)}$, and the interim analysis for the safety and the efficacy at both of dosing regimens in Japanese subjects in Study CA180138. The E-R analysis demonstrated that probability of the efficacy (MCyR) increased with increasing $\mathrm{C}_{\text {avg ss }}$ and decreasing the dose interruption duration. For the safety, higher $\mathrm{C}_{\min }$ ss were associated with more frequent key side effect, pleural effusion. No clinically meaningful exposure data could be provided for the dosing selection at the initial NDA submission since it was premature to make a conclusive statement about the PK linearity in Japanese subjects and the potential difference in $\mathrm{C}_{\max }$ and AUC between Japanese and non-Japanese subjects due to the limited PK data in Japanese subjects. For the assessment of PK comparison between Japanese and non-Japanese, it would be important to assess the average individual exposure at steady-state rather than the individual exposure on specific days in the case of long-term use of a drug which shows IOV such as dasatinib. In contrast with non-compartmental analysis, the model-based approach employed in this analysis enables us to utilize 
the integrated dasatinib concentration data including sparse samples across Japanese clinical trials, more appropriately evaluate PK linearity in larger sample size, and provide average individual PK parameter estimates after accounting for IOV. Adding the dose effect to $\mathrm{CL} / \mathrm{F}$ increased DIC value and its $95 \% \mathrm{CI}$ included zero, suggesting that dasatinib PK does not deviate from dose linearity, and therefore, $\mathrm{C}_{\min }$ ss at $100 \mathrm{mg}$ QD would be lower than that at $50 \mathrm{mg}$ BID with comparable $\mathrm{C}_{\text {avg ss }}$ between two regimens in Japanese subjects, which could offer more favorable safety profiles regarding pleural effusion at $100 \mathrm{mg}$ $\mathrm{QD}$ compared to $50 \mathrm{mg}$ BID without difference of the efficacy between the two dosing regimens in Japanese imatinib-resistant or -intolerant chronic phase CML patient population, based on the result of the E-R analysis. Although sample size is small, the result of Study CA180-138 in Japanese imatinib-resistant or -intolerant chronic phase CML subjects also supported the preferable safety at $100 \mathrm{mg}$ QD compared to $50 \mathrm{mg}$ BID. Adverse events of pleural effusion were reported from 1 of 11 subjects and 4 of 12 subjects at $100 \mathrm{mg}$ QD and $50 \mathrm{mg}$ BID, respectively, with the high efficacy at both of dosing regimens in Study CA180-138 ${ }^{35}$. In addition, the distribution of dasatinib exposure in Japanese subjects is similar to those of non-Japanese subjects after accounting for IOV (Fig. 6) in this PPK analysis indicated that no adjustment of the dasatinib dose is warranted for Japanese CML and $\mathrm{Ph}^{+}$ALL patients. Slightly higher Cminss was observed at $100 \mathrm{mg}$ QD in Japanese subjects than that in the non-Japanese subjects and the impact of the difference on the safety is unknown because of limited Japanese subjects at the dosage. However, dasatinib appeared to have the acceptable and tolerable safety profile at $100 \mathrm{mg}$ QD in Japanese imatinib-resistant or -intolerant chronic phase CML subjects in Study CA180-138 $8^{35)}$, and the post marketing surveillance of dasatinib in Japan may provide the supportive data for the safety profiles in Japanese patient populations in the future.

In conclusion, the concentration-time data of dasatinib in Japanese subjects with imatinib-resistant or -intolerant $\mathrm{CML}$ and $\mathrm{Ph}^{+} \mathrm{ALL}$ were well described by a linear 2-compartment model with first-order absorption. The PPK model contained the IOV on $F_{R}$ to account for between-visit difference of dasatinib exposure within a subject which was observed in Study CA180-031. Investigation of covariates includ- ing visual screening assessment and covariate modeling revealed neither marked trends nor clinically relevant effect on $\mathrm{CL} / \mathrm{F}$. CL/F was not significantly related to dasatinib dose and no food effect was found on $F_{R}$ in Japanese subjects. The PPK model of dasatinib in Japanese subjects is identical with that in corresponding non-Japanese subjects, and comparable exposures of Japanese subjects with that of nonJapanese subjects are obtained after accounting for IOV. This population PK analysis demonstrated that no dose adjustment is warranted for Japanese CML and $\mathrm{Ph}^{+}$ALL patients, their body weight, age, or gender, and illustrates the utility of modeling approach in drug development programs, especially for drugs showing IOV, for the assessment of PK linearity and the further comparison of drug exposure between different populations.

\section{Acknowledgements}

This work was carried out with the cooperation of many investigators from Japanese clinical trials, with a grant from Bristol-Myers Squibb Company (BMS). Special thanks go to Kazuhito Yamamoto, Hisashi Sakamaki, Masaya Okada, Masafumi Taniwaki, Ken-ichi Ishizawa. We have to thank their professional and energetic support helping us conduct Japanese clinical trials.

Our most sincere thanks go to our colleague and advisor of pharmacometrics group of R \& D, Shelly Wang and Amit Roy. We thank them for their guidance, encouragement and support during this work. We also want to express sincere gratitude to Shintaro Onishi, who led to the dasatinib development in Japan, for his support helping us in data collection and sharing information on related experimental details.

\section{Conflict of Interest}

No potential conflicts of interest relevant to this article were present.

\section{References}

1) Sprycel $^{\circledR}$ (Dasatinib Tablets) Japan Package Insert, BristolMyers KK, Tokyo, Japan. Revised : 06/2011.

2) Carter TA, Wodicka LM, Shah NP, Velasco AM, Fabian MA, Treiber DK, et al. Inhibition of drug-resistant mutants of ABL, KIT, and EGF receptor kinases. Proc Natl Acad Sci USA. 2005 ; 102(31) : 11011-6.

3) Lombardo LJ, Lee FY, Chen P, Norris D, Barrish JC, Behnia K, et al. Discovery of N-(2-chloro-6-methyl-phenyl)-2-(6-(4-(2hydroxyethyl) - piperazin-1-yl) -2-methylpyrimidin-4-ylamino) thiazole-5-carboxamide (BMS-354825), a dual Src/Abl kinase inhibitor with potent antitumor activity in preclinical assays. $J$ Med Chem. 2004 ; 47 (27) : 6658-61.

4) Shah NP, Tran C, Lee FY, Chen P, Norris D, Sawyers CL. Overriding imatinib resistance with a novel ABL kinase inhibitor. Science. $2004 ; 305(5682)$ : 399-401. 
5) Tokarski JS, Newitt JA, Chang CY, Cheng JD, Wittekind M, Kiefer SE, et al. The structure of Dasatinib（BMS-354825) bound to activated ABL kinase domain elucidates its inhibitory activity against imatinib-resistant ABL mutants. Cancer Res. 2006 ; 66 (11) : 5790-7.

6) Talpaz M, Shah NP, Kantarjian H, Donato N, Nicoll J, Paquette R, et al. Dasatinib in imatinib-resistant Philadelphia chromosomepositive leukemias. $N$ Engl J Med. $2006 ; 354(24): 2531-41$.

7) Cortes J, Rousselot P, Kim DW, Ritchie E, Hamerschlak N, Coutre S, et al. Dasatinib induces complete hematologic and cytogenetic responses in patients with imatinib-resistant or intolerant chronic myeloid leukemia in blast crisis. Blood. 2007 ; 109 (8) : 3207-13.

8) Hochhaus A, Kantarjian HM, Baccarani M, Lipton JH, Apperley JF, Druker BJ, et al. Dasatinib induces notable hematologic and cytogenetic responses in chronic phase chronic myeloid leukemia after failure of imatinib therapy. Blood. $2007 ; 109$ (6) : 2303-9.

9) Guilhot F, Apperley J, Kim DW, Bullorsky EO, Baccarani M, Roboz GJ, et al. Dasatinib induces significant hematologic and cytogenetic responses in patients with imatinib-resistant or intolerant chronic myeloid leukemia in accelerated phase. Blood. $2007 ; 109(10): 4143-50$

10) Quintas-Cardama A, Kantarjian H, Jones D, Nicaise C, O'Brien $S$, Giles F, et al. Dasatinib (BMS-354825) is active in Philadelphia chromosome-positive chronic myelogenous leukemia after imatinib and nilotinib (AMN107) therapy failure. Blood. 2007 ; 109(2) : 497-9.

11) Sakamaki H, Ishizawa K, Taniwaki M, Fujisawa S, Morishima $Y$, Tobinai K, et al. Phase $1 / 2$ clinical study of dasatinib in Japanese patients with chronic myeloid leukemia or Philadelphia chromosome-positive acute lymphoblastic leukemia. Int $J$ Hematol. $2009 ; 89(3): 332-41$.

12) Okada M, Yamamoto K, Sakamaki H, Kobayashi S, Ando K, Nakagawa Y, et al. Phase 2 study of dasatinib in patients with chronic phase CML-100 mg QD or $50 \mathrm{mg}$ BID. Jap J Clin Hematol. $2008 ; 49$ (9) : 256.

13) Porkka, K, Khoury HJ, Paquette RL, Matloub Y, Sinha R, Cortes JE. Dasatinib $100 \mathrm{mg}$ once daily minimizes the occurrence of pleural effusion in patients with chronic myeloid leukemia in chronic phase and efficacy is unaffected in patients who develop pleural effusion. Cancer. $2010 ; \mathbf{1 1 6}(2): 377-86$.

14) Shah NP, Kim DW, Kantarjian H, Rousselot P, Llacer PE, Enrico A, et al. Potent, transient inhibition of BCR-ABL with dasatinib $100 \mathrm{mg}$ daily achieves rapid and durable cytogenetic responses and high transformation-free survival rates in chronic phase chronic myeloid leukemia patients with resistance, suboptimal response or intolerance to imatinib. Haematologica. $2010 ; 95$ (2) : 232-40.

15) Kantarjian H, Cortes J, Kim DW, Dorlhiac-Llacer P, Pasquini R, DiPersio J, et al. Phase 3 study of dasatinib $140 \mathrm{mg}$ once daily versus $70 \mathrm{mg}$ twice daily in patients with chronic myeloid leukemia in accelerated phase resistant or intolerant to imatinib : 15-month median follow-up. Blood. 2009 ; 113 (25) : 6322-9.

16) Saglio G, Hochhaus A, Goh YT, Masszi T, Pasquini R, Maloisel F, et al. Dasatinib in imatinib-resistant or imatinib-intolerant chronic myeloid leukemia in blast phase after 2 years of followup in a phase 3 study : efficacy and tolerability of 140 milligrams once daily and 70 milligrams twice daily. Cancer. 2010 ; 116(16) :
3852-61.

17) Lilly MB, Ottmann OG, Shah NP, Larson RA, Reiffers JJ, Ehninger G, et al. Dasatinib $140 \mathrm{mg}$ once daily versus $70 \mathrm{mg}$ twice daily in patients with Ph-positive acute lymphoblastic leukemia who failed imatinib : Results from a phase 3 study. Am J Hematol. $2010 ; 85$ (3) : 164-70.

18) Kantarjian H, Shah NP, Hochhaus A, Cortes J, Shah S, Ayala M, et al. Dasatinib versus imatinib in newly diagnosed chronicphase chronic myeloid leukemia. N Engl J Med. 2010 ; 362 (24) : 2260-70.

19) Cortes JE, Jones D, O’Brien S, Jabbour E, Ravandi F, Koller C, et al. Results of dasatinib therapy in patients with early chronicphase chronic myeloid leukemia. J Clin Oncol. 2010 ; 28(3) : 398404.

20) Sprycel $^{\circledR}$ (Dasatinib Tablets) U.S. Package Insert, BristolMyers Squibb Company, Princeton, NJ. Revised : 10/2010.

21) Dai G, Pfister M, Blackwood-Chirchir A, Roy A. Importance of characterizing determinants of variability in exposure : application to dasatinib in subjects with chronic myeloid leukemia. $J$ Clin Pharmacol. 2008 ; 48(11) : 1254-69.

22) Nicaise C, Wang X, Roy A, Pfister M, Chen T, Bleickardt E, et al. Dasatinib pharmacokinetics and exposure-response (E-R): relationships to efficacy and safety in patients with chronic myelogenous leukemia in chronic phase (CML-CP). EHA congress 2008: Abstract 0559. URL http://www.eventure-online. com/eventure/publicSession.do?id=84530.

23) Beal S, Sheiner LB, Boeckmann A, Bauer RJ. NONMEM User's Guides. (1989-2009), Icon Development Solutions, Ellicott City, MD, USA, 2009.

24) Davidian M, Giltinan DM. Nonlinear models for repeated measurement data: an overview and update. J Agric Biol Environ Stat. $2003 ; 8(4)$ : 387-419.

25) Tsuchiwata S, Mihara K, Yafune A, Ogata H. Evaluation of Bayesian estimation of pharmacokinetic parameters. Ther Drug Monit. $2005 ; 27$ (1) : 18-24.

26) Ogata H (Eds). Clinical Pharmacokinetics, Second Edition. Maruzen Co, Ltd. 2007 ; 101-4. (in Japanese)

27) Furutani T. Bayes Statistical Data Analysis. Asakura Publishing Co, Ltd. $2008: 86$. (in Japanese)

28) Ando T. Bayes Statistical Modeling. Asakura Publishing Co, Ltd. $2010: 66-8$. (in Japanese)

29) Plummer M, Best N, Cowles K, Vines K. coda: Output analysis and diagnostic for MCMC. 2011 : 16. R package version 0.14-4. URL http://cran.r-project.org/web/packages/coda/coda.pdf

30) Cockcroft, Gault MH. Prediction of creatinine clearance from serum creatinine. Nephron. $1976: \mathbf{1 6}(1): 31-41$.

31) Ando T. Bayes Statistical Modeling. Asakura Publishing Co, Ltd. $2010: 168-9$ (in Japanese)

32) Spingelhalter DJ, Best NG, Carlin BP, van der linda A. Bayesian measures of model complexity and fit (with discussion and rejoinder). Journal of the Royal Statistical Society. 2002 ; B64 : 583-639.

33) MRC Biostatistics Unit, The BUGS Project. URL http://www. mrc-bsu.cam.ac.uk/bugs/winbugs/dicpage.shtml

34) Savic RM, Karlsson MO. Importance of shrinkage in empirical bayes estimates for diagnostics : ploblems and solutions. AAPSJ. $2009 ; 11(3): 558-69$.

35) Yamamoto K, Okada M, Kobayashi Y, et al. Phase II study of dasatinib $100 \mathrm{mg}$ daily administration in chronic phase CML Long Term Results-. JSH congress 2009 : Abstract No. 35. 